\title{
Los motores de la productividad en España: el caso del capital humano*
}

\author{
Laura Hernández \\ Instituto Valenciano de Investigaciones Económicas (Ivie) \\ Lorenzo Serrano \\ Universitat de València e Ivie
}

\section{Resumen}

Este trabajo analiza la contribución del capital humano al crecimiento de la productividad en España durante la reciente expansión y la actual crisis. El análisis utiliza técnicas de contabilidad del crecimiento y microdatos individuales y se centra en el impacto de los problemas de sobreducación de los trabajadores con formación superior y de una excesiva temporalidad. Los resultados indican la importancia de mitigar esos problemas a fin de garantizar un desarrollo sostenible. Las estimaciones obtenidas indican que los ajustes emprendidos durante la crisis van en esa dirección, confirmando la conveniencia de tomar medidas para mantener ese patrón tras esta crisis.

Palabras clave: productividad, sobreducación, capital humano, temporalidad.

Clasificación JEL: J24, J31, O40.

\begin{abstract}
This paper analyzes the contribution of human capital to productivity growth in Spain during the recent expansion and the current crisis. The analysis uses growth accounting techniques and individual microdata and focuses on the impact of overeducation problems of workers with higher education and excessive temporality. The results indicate the importance of mitigating these two problems to ensure a sustainable development. The resulting estimates indicate that adjustments are undertaken during the crisis in that direction, confirming convenience of taking measures to maintain that pattern after this crisis.
\end{abstract}

Keywords: productivity, overeducation, human capital, temporary workers.

JEL classification: J24, J31, O40.

\section{Introducción}

A lo largo de los últimos años España atravesó, en primer lugar, uno de los periodos de mayor crecimiento sostenido de su historia para, a continuación, verse inmersa en una de sus crisis más profundas. Durante el primer periodo, los aumentos de la producción y del empleo fueron muy superiores a los de la mayoría del resto de países de la Unión Europea. Por el contrario, desde el arranque de la actual crisis, el comportamiento de España ha sido mucho peor al de la mayoría, especialmente en lo que respecta al empleo.

* Lorenzo Serrano desea agradecer la ayuda recibida del Ministerio de Ciencia e Innovación (proyecto ECO2011-23248). 
Uno de los rasgos de la fase de crecimiento fue la mejora sostenida de los niveles de formación educativa de la población española. Esta mejora del capital humano, que afectó también de modo sustancial a la población ocupada, tendría que haber repercutido en la productividad. Los aumentos en el empleo y el PIB deberían de haberse visto acompañados por importantes mejoras en la productividad del trabajo. Sin embargo, de modo un tanto desconcertante, el comportamiento de la productividad fue poco satisfactorio, comparándose desfavorablemente con el seguido por el resto de países de nuestro entorno (Mas y Robledo, 2010; Mas y Stehrer, 2011; Cuadrado-Roura y Maroto, 2012).

Así pues, la mejora educativa en España, pese al contexto de crecimiento vigoroso en el que se produjo, no se tradujo, aparentemente, en mayores aumentos de la productividad. Esto sugiere la necesidad de profundizar en el análisis de la relación entre formación educativa y productividad. Las lecciones que se deriven de dicho análisis tienen relevancia no solo para comprender la evolución reciente de la economía española, sino también con vistas definir los riesgos y oportunidades de cara al futuro, una vez superada la actual crisis.

Este trabajo se estructura en 6 apartados con la presente introducción. El apartado 2 repasa el proceso de mejoras educativas en España. El apartado 3 examina la evolución de la sobreducación y la temporalidad como aspectos problemáticos en la relación entre capital humano, productividad y crecimiento. El apartado 4 describe la metodología empleada y los aspectos relativos al análisis empírico del caso español durante el periodo 2002-2010. El apartado 5 discute los resultados obtenidos. Finalmente, el apartado 6 presenta las principales conclusiones obtenidas.

\section{Las mejoras educativas en España}

La mejora de los niveles de educación completados por los trabajadores españoles ha sido sustancial durante los últimos años. El porcentaje de ocupados con estudios universitarios, un indicador habitual de las dotaciones de capital humano per cápita, pasó del 15,5 por 100 en 1995 al 26 por 100 en 2010 (Gráfico 1). Se trata de un aumento de más de 10 puntos porcentuales, equivalentes a un crecimiento del 67 por 100 en términos acumulados. Prácticamente la mitad de los nuevos ocupados durante el periodo 1995-2010 correspondió a ese tipo de estudios, poniendo de manifiesto el cambio experimentado durante esos años.

Los datos anteriores se refieren a lo que sucede con el crecimiento del colectivo más formado, pero dan una idea bastante ajustada de lo sucedido a nivel global. Examinando la evolución de los años medios de estudios de la población ocupada, un indicador sintético de la situación global en términos de formación educativa, se observa también (gráfico 1) una mejora sostenida. Los años medios pasan de nueve en 1995 a once en 2010, con un crecimiento acumulado del 23 por 100. 


\section{GRÁFICO 1}

FORMACIÓN EDUCATIVA DE LA POBLACIÓN OCUPADA (1995=100)

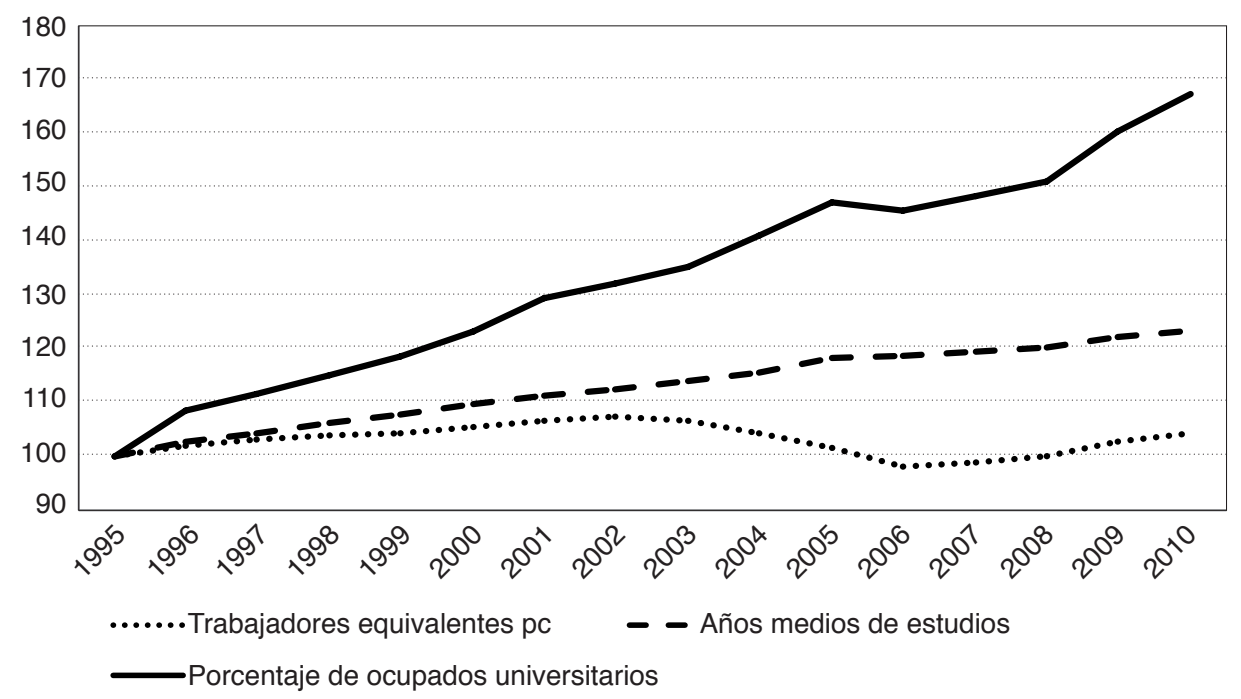

FUENTE: INE y Bancaja-Ivie.

A esa mejora de los niveles educativos han contribuido factores como la generalización del acceso a la educación primaria obligatoria y gratuita de los seis a los catorce años lograda de modo efectivo con la aplicación de la Ley General de Educación de 1970. A partir de ese momento y durante las siguientes décadas la elevación de la renta per cápita y un impulso decidido de la oferta educativa por parte del sector público (en todos los niveles de enseñanza, obligatorios y no obligatorios) ha continuado alimentando el proceso. La ampliación de la escolaridad obligatoria a comienzos de los noventa hasta los dieciséis años de edad forma parte de ese esfuerzo de la política educativa, con resultados quizá más discutibles en términos de calidad de la educación.

Esta evolución debería haber tenido un efecto positivo apreciable sobre la productividad de acuerdo a la teoría del capital humano, que establece una relación directa entre inversión en capital humano y crecimiento económico. Desde su origen esta teoría consideró al capital humano como un factor explicativo clave de la diferencia entre aumentos de la producción y aumentos de la cantidad de factores productivos utilizados (Schultz, 1960). Posteriormente los modelos teóricos de crecimiento económico han incorporado al capital humano como uno de sus elementos centrales, postulándolo frecuentemente como el principal motor de crecimiento, tanto si se trata de modelos de crecimiento endógeno (Lucas, 1988; Romer, 1990) como si se trata de modelos neoclásicos (Mankiw et al., 1992). 
En primer lugar, el capital humano contribuiría al crecimiento económico impulsando la productividad del trabajo de modo semejante a como sucede con otros tipos de capital, como el capital físico. Además, también contribuiría al crecimiento mediante el impulso de la innovación e imitación tecnológica y su efecto en el ritmo de progreso técnico (Nelson y Phelps, 1970; Welch, 1970).

Existe evidencia empírica sobre el efecto positivo del capital humano en el crecimiento económico y la productividad en el caso español. Diversos trabajos obtienen un efecto positivo del capital humano a largo plazo en la economía española, durante el periodo que va de la década de los sesenta a la de los noventa (Serrano, 1999; De la Fuente 2002; Sosvilla-Rivero y Alonso-Meseguer 2005; Pablo-Romero y Gómez-Calero, 2008).

Por otra parte, algún estudio reciente (Lacuesta et al., 2011) indica que las mejoras educativas serían en buena medida solo aparentes y podrían no haber dado lugar a mayores dotaciones del capital humano per cápita en los últimos tiempos en España. Por el contrario, según estos autores, el capital humano per cápita incluso habría disminuido ligeramente durante el periodo 1996-2006, una vez se consideran aspectos como las ocupaciones desarrolladas, la caída de los rendimientos de la educación y la capacidad innata de los trabajadores. Según los autores esto estaría ligado a problemas de sobreducación y a la contratación de individuos especialmente poco productivos que se habría producido durante el último boom económico y la fortísima creación de empleo que lo acompañó.

Carrasco et al. (2011) utilizan regresiones cuantílicas para explicar el pobre comportamiento de los salarios reales durante el periodo 1995-2006. Sus resultados indican que esa evolución se debería especialmente a la caída de los rendimientos de la educación y la experiencia laboral en los deciles elevados de la distribución salarial, y no tanto a cambios en las características de los trabajadores y los puestos de trabajo (sus resultados indican que, en términos de calidad del trabajo, las mejoras en composición por educación y experiencia más que compensarían el efecto negativo de la inmigración o el crecimiento de los puestos de trabajo poco cualificados). Estos autores señalan como responsables al desajuste entre las cualificaciones de los trabajadores y las requeridas por los puestos de trabajo. Se trataría de un problema ligado al deficiente funcionamiento del sistema educativo y a aspectos institucionales del mercado de trabajo español, caracterizado por la dualidad y el exceso de temporalidad y de rotación. Esto último dificultaría el aprovechamiento de la formación de los trabajadores con mayores niveles educativos.

Existen, por tanto, serias dudas sobre la contribución de las mejoras educativas observadas en el caso español. La mera cantidad de educación podría ser, hasta cierto punto, un indicador engañoso del capital humano, tal y como sugieren los resultados del análisis del crecimiento regional en Serrano (2012). El Gráfico 1 muestra también la evolución de un tercer indicador de capital humano per cápita que refleja a cuántos trabajadores sin formación ni experiencia equivale el ocupado promedio. Este indicador considera las características personales de los individuos, incluyendo el nivel educativo más elevado alcanzado y otros aspectos como el sexo 
y la edad (que es un indicador aproximado de la experiencia laboral). El indicador utiliza la valoración que el mercado hace de todas esas cualidades en términos del salario relativo correspondiente (Serrano y Soler, 2010).

Como puede observarse, el crecimiento acumulado de ese indicador durante el periodo 1995-2010 es la cuarta parte que el de los años medios de estudios, por no hablar de las diferencias cuando se compara con el comportamiento del porcentaje de ocupados universitarios (que crece dieciséis veces más deprisa durante el periodo).

La comparación entre esos diversos indicadores resulta especialmente llamativa a partir de 2002. Mientras los años medios o el porcentaje de universitarios siguen creciendo a un ritmo rápido y similar al de periodos previos, el indicador de trabajadores equivalentes no solo modera su crecimiento, sino que cae hasta 2006, situándose durante el periodo 2006-2008 por debajo del nivel de 1995. Es precisamente durante el periodo de crisis cuando ese indicador crece a un ritmo similar al de los años de estudios.

Esta evidencia muestra la existencia durante los años de expansión de notables dificultades para convertir los aumentos de los niveles de formación de los trabajadores en mayor productividad y mejores salarios. También apunta a que esas dificultades estarían remitiendo durante el periodo más reciente, en especial durante el periodo de crisis.

\section{Algunos sospechosos habituales: sobreducación y contratos temporales}

Dos rasgos característicos del mercado de trabajo español son considerados de modo habitual como responsables de buena parte de los problemas de productividad de nuestra economía. En primer lugar, la generalización de situaciones de desajuste educativo en que trabajadores con elevados niveles educativos desarrollan ocupaciones que no exigen esos niveles de cualificación y, por tanto, pueden considerarse como sobreducados en relación a los requerimientos del puesto de trabajo. En segundo lugar, la fuerte dualidad del mercado laboral, con un uso muy intensivo de los contratos temporales con carácter estructural y en todo tipo de actividades.

Ambos fenómenos pueden tener un importante impacto en la evolución de la productividad y serán considerados más tarde en el análisis empírico a fin de tratar de cuantificar su efecto en la economía española ${ }^{1}$.

${ }^{1}$ Durante el periodo analizado ha habido otros cambios importantes en la composición de la fuerza laboral, como por ejemplo la masiva llegada de inmigrantes a nuestro país o la incorporación de la mujer al mercado de trabajo. El género es, precisamente, uno de las dimensiones utilizadas para definir los tipos de trabajo. Se trata de cuestiones no contempladas explícitamente en sí mismas en este trabajo más allá de su impacto a través de la composición por ocupaciones y tipos de contrato. En cualquier caso, se trata de dos colectivos especialmente afectados por esos dos factores. 


\subsection{La sobreducación en España}

Mediante la inversión en educación las personas adquieren competencias y conocimientos productivos, incrementando su capital humano y su capacidad productiva. Se trata precisamente de uno de los principales motivos que llevan a los individuos a prolongar su formación educativa más allá de la enseñanza obligatoria. Así esperan lograr una mejor carrera profesional con mejores salarios, acordes a su mayor capital humano.

Ahora bien, que todo eso se materialice depende de que ese capital humano sea utilizado apropiadamente. Sin embargo, cuando se producen problemas de mala asignación, ese resultado deseado y deseable no se da. Eso es lo que sucede en las situaciones de sobreducación. Cuando los trabajadores mejor formados se emplean en sectores y ocupaciones en que no hace falta esa formación educativa no cabe esperar esas ventajas en términos de mayor productividad, al menos no en la medida deseable. La literatura sobre este tema muestra la relevancia de este fenómeno en el caso de España y su creciente intensidad (véase, por ejemplo, Alba-Ramírez, 1993; Budría y Moro-Egido, 2008; García-Montalvo y Peiró, 2009, o Hernández y Serrano, 2012).

Hay que señalar que la sobreducación no es una cuestión sencilla a la hora de pasar a las definiciones concretas para el análisis empírico. El problema de la correspondencia entre la formación del trabajador y aquella formación requerida por el puesto de trabajo ocupado es complicado. A menudo se carece de información estadística suficientemente detallada. En Hernández y Serrano (2012) se discute esa problemática.

En nuestro caso vamos a definir las situaciones de desajuste educativo a partir de correspondencias entre niveles educativos del individuo (a partir de la ISCED-97, concretada en el caso español en la CNED-2000) y grupos de ocupaciones (a partir de la ISCO-88, concretada en el caso español en la CNO-94). Precisamente un ejemplo de lo complejo de esta cuestión es que la reciente introducción en la Unión Europea de la nueva ISCO-2008 ha supuesto un cambio en las ocupaciones incluidas en los diferentes categorías, con notable impacto en los resultados para muchos países, y también en España, donde significa una notable caída en el peso de la ocupaciones consideradas cualificadas respecto a lo que venía siendo tradicional.

Un indicador muy habitual de ese tipo, ampliamente utilizado por organizaciones como la OCDE o la propia Unión Europa, es el porcentaje de trabajadores con estudios superiores (sean universitarios o de formación profesional superior) en ocupaciones correspondientes a los grupos 4-9 de la clasificación de ocupaciones. La idea es que son los puestos directivos, científicos, técnicos y profesionales (incluidos en los grupos 1-3 de la clasificación de ocupaciones) los que propiamente requieren formación de tipo superior, mientras que el resto pueden desempeñarse perfectamente con niveles de formación media o básica.

El Gráfico 2 muestra la evolución de ese indicador desde 1995 en España y en el conjunto de la Unión Europea. En primer lugar, se observa que una parte muy relevante de los trabajadores con estudios superiores realiza ocupaciones que implican una situación de sobreducación. En 2010 casi un 33 por 100 de los ocupados con estudios 


\section{GRÁFICO 2}

\section{TRABAJADORES CON ESTUDIOS SUPERIORES EN OCUPACIONES DESAJUSTADAS}

(En \%)

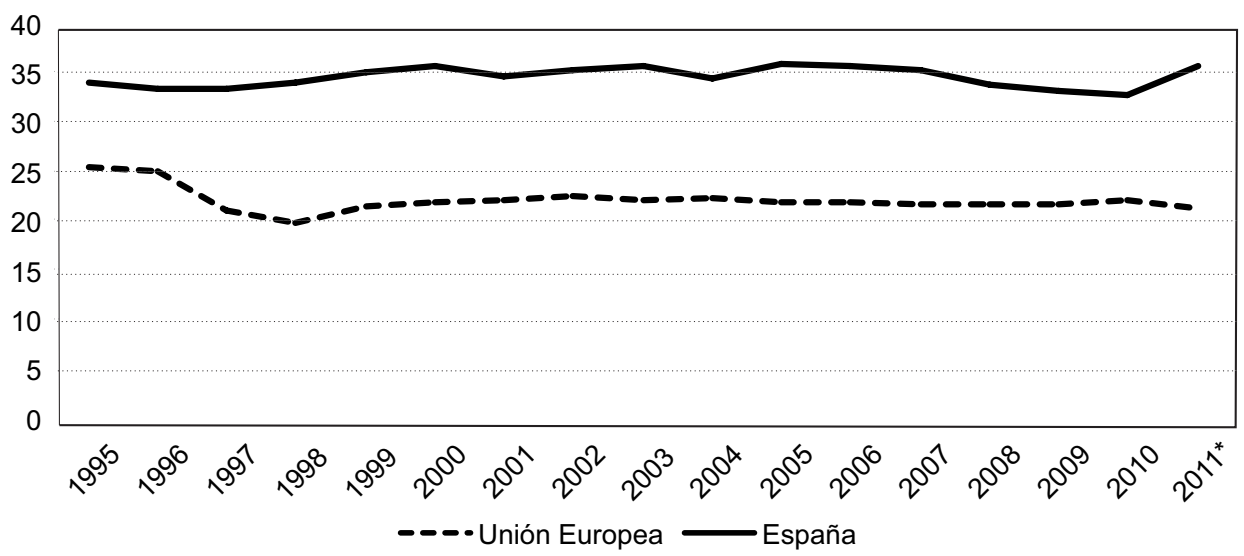

NOTA: * Cambio metodológico en la clasificación de ocupaciones (paso de la CNO-94 a la CNO-08). FUENTE: EUROSTAT.

superiores estaría desajustado, cifra que con los criterios de la nueva clasificación de ocupaciones aplicada en 2011 llegaría al 35,6 por 100. Además, se trata de un fenómeno permanente, ya que el porcentaje de sobreducación se sitúa en España por encima del 33 por 100 durante prácticamente todo el periodo contemplado. En todo caso se aprecia una cierta tendencia creciente durante el periodo de expansión económica, seguida por un descenso moderado a partir de la crisis.

En segundo lugar, resulta especialmente llamativa la diferencia de comportamiento respecto al patrón que predomina en la Unión Europea, donde el porcentaje de sobreducación se mantiene relativamente estable desde finales del siglo pasado en valores ligeramente por encima del 20 por 100 .

Esos datos ponen de manifiesto el grave problema de sobreducación que sufre España, su carácter fuertemente estructural y su intensificación durante los años del boom. Todo esto debería haber afectado de modo negativo a los niveles de productividad, frenando su crecimiento antes de la crisis. Por otra parte, la media europea también debe servir de referencia acerca de las mejoras en términos de sobreducación que podrían ser consideradas razonables. Así, una reducción de la sobreducación en un 40 por 100 situaría a España en torno a la media europea. Se trata de una mejora ya muy exigente en sí misma. Progresos más sustanciales serían progresivamente más complicados de aceptar como factibles a corto y medio plazo. 


\subsection{La temporalidad}

Otra característica particular del mercado de trabajo español que podría contribuir a frenar las ganancias de productividad es la gran presencia de contratos temporales. Se ha insistido tanto en sus aspectos negativos que conviene recordar el origen de los cambios legislativos que ha acabado motivando ese uso desproporcionado de los contratos temporales.

La crisis del petróleo de finales del siglo pasado se conjugó en el caso de España con un mercado de trabajo extraordinariamente rígido para provocar tasas de desempleo que se acercaron al 25 por 100 a mediados de los ochenta, cifras anormales y alejadas de lo sucedido en el resto de economías desarrolladas. La respuesta de política económica consistió en una serie de reformas del mercado de trabajo caracterizadas por introducir flexibilidad a través de los contratos de los nuevos ocupados, manteniendo al mismo tiempo las condiciones de los ya empleados. Un instrumento fundamental fue la figura de los contratos temporales, caracterizados por costes de despido menores. El resultado positivo de esas reformas fue su impulso al crecimiento del empleo, pero supuso un porcentaje de contratos temporales muy elevado.

El Gráfico 3 indica que a lo largo del periodo de expansión entre un 30 por 100 y un 35 por 100 de los asalariados tenía un contrato temporal. Esos valores están muy por encima de lo habitual en el resto de economías de la UE, donde la media osciló entre el 10 por 100 y el 15 por 100 . Hay que tener en cuenta que la temporalidad fue todavía más acusada durante esos años en el caso del sector privado español, con

\section{GRÁFICO 3}

ASALARIADOS CON CONTRATO TEMPORAL

(En \%)

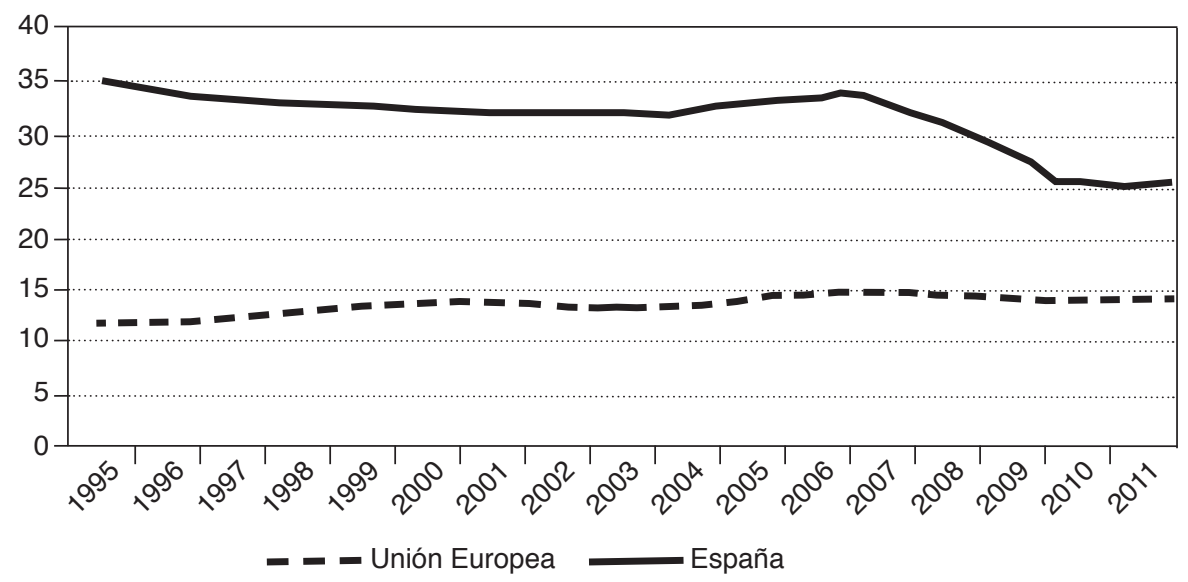

FUENTE: EUROSTAT. 
porcentajes sistemáticamente superiores al 35 por 100. Se trata, pues, de un rasgo muy específico del mercado de trabajo español y, de hecho, solo Polonia o Portugal presentan niveles comparables.

La crisis supone un cambio radical en este ámbito. En España se inicia un descenso del porcentaje de temporalidad asociado al gran ajuste de empleo. La crisis supone para las empresas la exigencia de reducir su producción y también sus costes laborales. En el caso español la respuesta se realiza poco a través de la flexibilidad de los tiempos de trabajo o los salarios y se produce, preferentemente, mediante las reducciones de plantilla. El ajuste del empleo temporal constituye la forma menos costosa de reducir plantillas de modo rápido en las fases iniciales de la crisis, por lo cual la caída del empleo se concentra, al menos durante esa fase, en el empleo temporal.

Paradójicamente ese objetivo tanto tiempo perseguido, la reducción de la temporalidad, se produce finalmente pero del modo menos deseable. No por el desarrollo de empleo indefinido que lo sustituya, sino por la concentración de la destrucción de empleo en ese colectivo. La temporalidad cae del 34 por 100 en 2006 hasta situarse en torno al 25 por 100, aunque la reducción se concentra en los primeros momentos de la crisis. A partir de 2009 el porcentaje se estabiliza, reflejando que la destrucción de empleo ya pasa a afectar con intensidad similar a asalariados temporales y fijos. Ese porcentaje del 25 por 100 sigue siendo sustancialmente mayor que la media de la UE, situada en el 14 por 100. Esa referencia significa que reducir la temporalidad en un 45 por 100 situaría a España en la media europea. De nuevo ese sería un cambio muy sustancial y descensos más acusados pueden considerarse como poco factibles a corto y medio plazo.

Una temporalidad tan fuerte $\mathrm{y}$, además, extendida durante un periodo tan prolongado como en el caso español tiene efectos negativos sobre la productividad. En primer lugar, genera demasiada rotación en los puestos de trabajo y eso daña a la productividad. Ni las empresas ni los trabajadores tienen incentivos para invertir en formación específica ligada al puesto de trabajo. En segundo lugar, la experiencia conseguida por el trabajador se pierde en gran medida al extinguirse la relación contractual y el proceso comienza de nuevo, para el trabajador en su nueva empresa, y para la empresa con su nuevo trabajador. Este problema puede no ser muy importante en ciertas actividades, pero la elevada temporalidad de España implica que el fenómeno también afectó en nuestro caso a las actividades donde esa cuestión sí resulta grave. Naturalmente, este tipo de problemas no ha tenido una repercusión uniforme, sino que ha afectado principalmente a los nuevos entrantes en el mercado de trabajo español: jóvenes e inmigrantes. De hecho, en el caso de los inmigrantes, también en el de los jóvenes menores de 30 años, se han mantenido tasas de temporalidad en torno al 60 por 100 durante buena parte del periodo contemplado. 


\subsection{Estructura de ocupaciones}

En alguna medida todos los problemas señalados están vinculados al tipo de tejido productivo de la economía española: empresas más pequeñas y en sectores más tradicionales, algo que se traduce en una menor importancia de las actividades que exigirían más cualificación y donde los trabajadores más formados serían más productivos. En definitiva, la sobreducación puede considerarse en cierto sentido como un problema de insuficiencia de puestos de trabajo en ocupaciones cualificadas. Considerando de nuevo las ocupaciones en los grupos 1-3 de la CNO como las que exigen una elevada cualificación, el gráfico 4 muestra la evolución de la estructura del empleo en España y en el conjunto de la $\mathrm{UE}^{2}$.

\section{GRÁFICO 4}

\section{OCUPACIONES CUALIFICADAS}

(En \%)

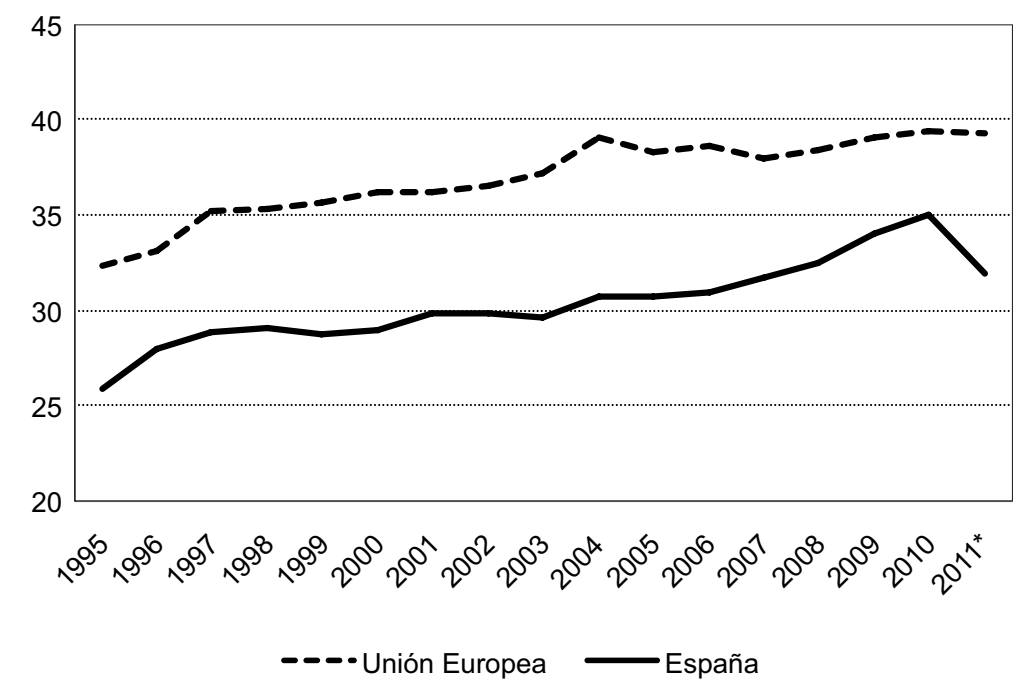

NOTA: * Cambio metodológico en la clasificación de ocupaciones (paso de la CNO-94 a la CNO-08)

FUENTE: EUROSTAT y elaboración propia.

${ }^{2}$ Excede el propósito de este trabajo analizar las razones de la evolución de la demanda de educación en términos de las ocupaciones que requieren más o menos nivel de cualificación. Se trata de una cuestión importante en relación a la que aspectos como el boom de la construcción o la llamada teoría de la polarización (según la cual, el desarrollo tecnológico debería hacer que se incrementara la demanda de ocupaciones de alto y bajo nivel de cualificación, mientras que la demanda de cualificaciones medias debería disminuir) pueden ser muy relevantes. 
Los datos confirman la escasa presencia de las ocupaciones más cualificadas en España. Nuestro país se sitúa permanentemente por detrás de la media europea, por no hablar de los niveles aún más elevados de algunos países concretos. Por otra parte, también puede apreciarse la mejora constante a lo largo del periodo, con una ganancia de nueve puntos porcentuales entre 1995 y 2010. Ese crecimiento se produce tanto durante el periodo de expansión económica como, y muy especialmente, tras la crisis. Todo parece indicar que los ajustes de plantilla y los cierres de empresas están afectando sobre todo a la parte menos productiva de nuestra economía, menos competitiva y, por tanto, en una situación más débil para afrontar la crisis. La época de bonanza permitió la creación de empresas y empleos poco sostenibles en un contexto más negativo para los negocios como el propiciado por la crisis.

El resultado es una progresiva convergencia a la estructura ocupacional típica de los países de la Unión Europea, aunque todavía persista una brecha sustancial respecto a ellos.

El aparente giro de la tendencia en 2011 debe atribuirse al cambio en la clasificación de ocupaciones utilizada por las agencias estadísticas homogéneas. Esto da lugar a un descenso en los empleos en ocupaciones consideradas cualificadas en el caso español, pero no modifica la evolución creciente durante el periodo analizado.

\section{Metodología}

Por las razones descritas en los apartados anteriores, el nivel educativo de un nuevo trabajador puede resultar un indicador impreciso del capital humano realmente incorporado al proceso productivo y, por tanto, de su efecto sobre la productividad.

La contabilidad del crecimiento constituye un marco analítico apropiado para tener en cuenta esas cuestiones de cara al problema que nos ocupa. Las técnicas habitualmente utilizadas por la contabilidad del crecimiento permiten estimar la contribución que la variación en el uso de cada factor productivo tiene en el aumento de la producción. Así, puede integrarse de modo natural el impacto de cambios en la composición interna de cada uno de los factores, en particular los referidos a variaciones en la calidad del trabajo.

Siguiendo la aproximación de Jorgenson, Gollop y Fraumeni (1987), el crecimiento de la producción entre dos periodos $t-1 \mathrm{y} t$ que cabe atribuir al factor trabajo, vendría dado por:

$$
\frac{W_{t}+W_{t-1}}{2} \sum_{i t} \frac{\omega_{t}+\omega_{i t-1}}{2}\left(\ln H_{i t}-\ln H_{i t-1}\right)
$$

donde $W_{t}$ es el peso de las rentas del trabajo en la renta total en el periodo $t, \omega_{i t}$ es el peso de las rentas de los trabajadores del tipo $i$ en las rentas del trabajo del periodo $t$ y $H_{i t}$ es el número de trabajadores de tipo $i$ en el periodo $t$. En definitiva, la contribución corresponde a la media ponderada de la tasas de crecimiento de cada tipo de trabajo, 
donde las ponderaciones reflejan el peso medio de las rentas laborales de cada tipo de trabajador en la renta total.

Es posible descomponer esa contribución global del factor trabajo en la parte que corresponde al aumento del número trabajadores y, por otra parte, en la contribución de los cambios en la calidad del trabajo debidos a variaciones en el tipo de trabajo utilizado. Se distingue así entre dos vías a través de las que el factor trabajo produce más: bien porque se trabaja más (cantidad) o bien porque se utilizan trabajadores más cualificados y productivos (calidad).

La contribución ligada al mero incremento de la cantidad de trabajo utilizada corresponde a:

$$
\frac{W_{t}+W_{t-1}}{2} \sum_{i t} \frac{\omega_{i t}+\omega_{i t-1}}{2}\left(\ln H_{t}-\ln H_{t-1}\right)=\frac{W_{t}+W_{t-1}}{2} \sum_{i t}\left(\ln H_{t}-\ln H_{t-1}\right)
$$

donde $H_{t}$ es el número total de trabajadores en el periodo $t$. Como puede apreciarse, la magnitud del efecto viene dada por la tasa de crecimiento del total de trabajadores ponderada por la participación de la rentas del trabajo en la renta total.

La contribución ligada a variaciones en la calidad del trabajo utilizado, debido a cambios en el tipo de trabajadores empleados, se obtiene como:

$$
\frac{W_{t}+W_{t-1}}{2} \sum_{i t} \frac{\omega_{i t}+\omega_{i t-1}}{2}\left(\frac{\ln H_{i t}}{\ln H_{t}}-\frac{\ln H_{i t-1}}{\ln H_{t-1}}\right)
$$

Así pues, el crecimiento de la calidad del trabajo (o capital humano per cápita) se estimaría como:

$$
\sum_{i t} \frac{\omega_{i t}+\omega_{i t-1}}{2}\left(\frac{\ln H_{i t}}{\ln H_{t}}-\frac{\ln H_{i t-1}}{\ln H_{t-1}}\right)
$$

En definitiva, el crecimiento del capital humano per cápita entre el periodo $t-1$ y el periodo $t$ se estima como una media ponderada de las tasas de crecimiento relativo de cada tipo de trabajo, donde las ponderaciones vienen dadas por el peso de los salarios de cada tipo de trabajo en las rentas del trabajo. El método agrega los diferentes tipos de trabajador en función de sus productividades relativas, productividades que se aproximan a través de los salarios de mercado.

En particular, es necesario observar que no se están utilizando valoraciones a priori del capital humano asociado a cada tipo de trabajador (e.g. años de estudio). Por el contario, la valoración que refleja el propio mercado de trabajo es el factor decisivo. De este modo, si un nivel educativo específico no supusiese realmente más capital humano, ni más productividad, la retribución salarial lo reflejaría y, por tanto, el indicador de calidad del trabajo no crecería.

Otra ventaja del método empleado es su flexibilidad a la hora de incorporar todas las variables que se consideren relevantes para la calidad del trabajo, así como 
para considerar tantos grupos como se desee dentro de cada una de esas variables. Las variables consideradas incluyen características personales (edad, sexo y nivel educativo), así como del puesto de trabajo (tipo de contrato, ocupación). El análisis hace referencia al caso de la población ocupada de entre 16 y 64 años. Se han considerado cinco cohortes de edad (16-24, 25-34, 35-44, 45-54 y 55-64); dos categorías de sexo; tres niveles de formación (hasta estudios obligatorios, estudios secundarios postobligatorios y estudios superiores); dos categorías por tipo de contrato (temporales y resto) y tres ocupaciones (de baja cualificación, grupo 9 de la CNO; de cualificación media, grupos 4-8 de la CNO, y de cualificación alta, grupos 1-3 de la CNO). Esto supone distinguir 180 tipos de trabajo.

Los datos de ocupados de entre 16 y 64 años por edad, nivel educativo completado, tipo de contrato, ocupación, sexo y nacionalidad proceden de los microdatos de las encuestas individuales de los segundos trimestres de la Encuesta de Población Activa (EPA) del Instituto Nacional de Estadística (INE). Los datos sobre salarios por edad, nivel educativo completado, tipo de contrato, ocupación, sexo y nacionalidad proceden de los microdatos de la Encuesta de Estructura Salarial cuatrienal del INE correspondientes a los años 2002 y $2006^{3}$.

Con el fin de obtener los pesos en la renta laboral de cada tipo de trabajo se han utilizado las estimaciones obtenidas de regresiones de ecuaciones salariales a partir de los datos de la Encuestas de Estructura Salarial de 2002 y 2006 (EES-2002 y EES2006). En concreto, se trata de ecuaciones salariales de tipo minceriano (Mincer, 1974).

Se han utilizado especificaciones donde se estimaba el coeficiente salarial para cada uno de los 180 tipos de trabajo y otras en las que se incluían por separado dummies de edad, sexo, tipo de contrato, ocupación y nivel de estudios completados como:

$$
\ln \left(W_{t j}\right)=\beta_{o}+\sum_{i} \beta_{i} E d u_{t j}+\Sigma_{a} \beta_{a} E_{d a d_{t j}}+\Sigma_{z} \beta_{3 z} C_{z t j}
$$

donde $W$ es el salario bruto del trabajador $j$ en el periodo $t, E d u$ es una dummy que muestra su nivel de formación educativa, Edad es una dummy que muestra su edad y $C$ es un vector de variables dummy que incluyen sexo, tipo de ocupación y tipo de contrato. El Cuadro 1 muestra los resultados para esta segunda especificación.

${ }^{3}$ Se ha utilizado una muestra especial de la EES 2006 a fin de que los datos fuesen comparables con los de la EES 2002. Esa muestra no incluye a los establecimientos de menos de 10 trabajadores y los sectores incluidos en ambas oleadas son homogéneos y corresponden únicamente a las secciones C-K y M-O de la CNAE-93. El uso de la EES 2006 para el periodo posterior a ese año supone, evidentemente, una limitación debida al carácter muy reciente de los datos de la EES 2010. Un examen preliminar de esa nueva información indica, sin embargo, que los resultados esencialmente se mantendrían, aunque se observa un cambio en la tendencia previa negativa del rendimiento de la educación y una menor penalización salarial relativa para los trabajadores temporales. 


\section{CUADRO 1}

ECUACIONES SALARIALES (AÑOS 2002 Y 2006)

\begin{tabular}{|c|c|c|c|c|c|c|}
\hline & \multicolumn{3}{|c|}{ EES-2002 } & \multicolumn{3}{|c|}{ EES-2006 } \\
\hline & Coef. & Std. Err. & $\mathbf{t}$ & Coef. & Std. Err. & $\mathbf{t}$ \\
\hline Mujer & $-0,210$ & 0,0031 & $-68,21$ & $-0,200$ & 0,0030 & $-65,78$ \\
\hline 25-34 años & 0,089 & 0,0045 & 19,59 & 0,084 & 0,0049 & 16,98 \\
\hline 35-44 años & 0,241 & 0,0048 & 50,38 & 0,211 & 0,0052 & 41,01 \\
\hline 45-54 años & 0,341 & 0,0055 & 62,21 & 0,304 & 0,0056 & 54,01 \\
\hline 55-64 años & 0,370 & 0,0069 & 53,33 & 0,357 & 0,0073 & 48,62 \\
\hline Extranjero & $-0,029$ & 0,0074 & $-3,98$ & $-0,042$ & 0,0050 & $-8,28$ \\
\hline Temporal & $-0,145$ & 0,0030 & $-48,73$ & $-0,118$ & 0,0031 & $-38,09$ \\
\hline ISCED 3-4 & 0,105 & 0,0044 & 24,08 & 0,097 & 0,0043 & 22,54 \\
\hline ISCED 5-6 & 0,238 & 0,0051 & 46,68 & 0,221 & 0,0044 & 50,46 \\
\hline CNO 1-3 & 0,519 & 0,0059 & 88,01 & 0,504 & 0,0053 & 94,84 \\
\hline CNO 4-8 & 0,156 & 0,0036 & 43,48 & 0,139 & 0,0034 & 40,49 \\
\hline Constante & 1,713 & 0,0052 & 332,04 & 1,836 & 0,0055 & 333,29 \\
\hline Observaciones & \multicolumn{3}{|c|}{201.897} & \multicolumn{3}{|c|}{174.685} \\
\hline F-test & \multicolumn{3}{|c|}{$4.470,13$} & \multicolumn{3}{|c|}{$3.390,98$} \\
\hline Prob $>F$ & \multicolumn{3}{|c|}{0} & \multicolumn{3}{|c|}{0} \\
\hline $\mathrm{R}^{2}$ & \multicolumn{3}{|c|}{0,4105} & \multicolumn{3}{|c|}{0,389} \\
\hline
\end{tabular}

NOTA: La variable dependiente es el logaritmo neperiano del salario por hora.

Los valores, que reflejan los $\beta$ estimados de la ecuación [5], han de interpretarse como las diferencias salariales relativas respecto al trabajador de referencia (hombre, español, con estudios obligatorios como máximo, que trabaja en una ocupación del grupo 9 -trabajadores no cualificados-, de 16 a 24 años de edad y con un contrato indefinido). Los resultados son los habituales en el análisis del caso español. Todas las diferencias de categoría resultan significativas e indican un salario creciente con la formación educativa alcanzada y la edad (indicador aproximado de experiencia laboral), y menor en el caso de mujeres, extranjeros y trabajadores con contrato temporal. Especialmente sustancial es la diferencia salarial asociada al tipo de ocupación, con un incremento destacable asociado a las ocupaciones que requieren una mayor cualificación (CNO 1-3, con un salario un 50 por 100 mayor todo lo demás constante).

Los resultados son cualitativamente similares en 2002 y 2006, aunque se observa un descenso del rendimiento salarial ligado a la edad (experiencia), al tipo de ocupación y también a la formación educativa alcanzada. La tendencia a la caída en el rendimiento de la educación concuerda con los resultados de trabajos anteriores 
(por ejemplo, Pastor et al., 2007; Felgueroso et al., 2010; Murillo et al., 2010; Raymond, 2011). Las diferencias entre contratos temporales e indefinidos parecen haberse atenuado entre esos dos años.

\section{Resultados}

Los resultados sobre la evolución de la calidad del trabajo se muestran en el Cuadro 2. El primer panel muestra las estimaciones teniendo en cuenta la evolución de los salarios relativos de acuerdo a los datos de la EES-2002 y de la EES-2006, considerando los primeros como representativos de la situación en 2002 y los segundos como representativos de la situación de 2006 en adelante.

\section{CUADRO 2}

CRECIMIENTO ANUAL ESTIMADO DE LA CALIDAD DEL TRABAJO (En \%)

\begin{tabular}{|c|c|c|c|}
\hline \multirow{2}{*}{} & \multicolumn{3}{|c|}{ Combinando datos de la EES-2002 y la EES-2006 } \\
\cline { 2 - 4 } & Años medios & $\mathbf{1 8 0}$ dummies & Ecuaciones salariales \\
\hline $2002-2006$ & 1,32 & 0,52 & 0,48 \\
\hline $2006-2010$ & 1,00 & 0,98 & 0,88 \\
\hline & \multicolumn{3}{|c|}{ Usando siempre salarios de la EES-2002 } \\
\hline & Años medios & $\mathbf{1 8 0}$ dummies & Ecuaciones salariales \\
\hline $2002-2006$ & 1,32 & 0,51 & 0,49 \\
\hline $2006-2010$ & 1,00 & 1,06 & 0,96 \\
\hline & \multicolumn{3}{|c|}{ Usando siempre salarios de la EES-2006 } \\
\hline & Años medios & $\mathbf{1 8 0}$ dummies & Ecuaciones salariales \\
\hline $2002-2006$ & 1,32 & 0,54 & 0,47 \\
\hline $2006-2010$ & 1,00 & 0,98 & 0,88 \\
\hline
\end{tabular}

En primer lugar, hay que señalar que los resultados obtenidos al contemplar 180 salarios diferentes son algo mayores, pero similares a los obtenidos a partir de ecuaciones como la ecuación [5]. Durante el periodo 2002-2006, perteneciente a la fase expansiva, la mejora estimada de la calidad del factor trabajo (en torno al 0,5 por 100 anual) queda muy lejos del crecimiento de las dotaciones educativas de los trabajadores (cuyos años medios de estudios crecían al 1,3 por 100 anual). Esto permite tener una idea de la magnitud del problema, ya que la contribución real del capital humano al crecimiento de la productividad en España habría sido muy inferior al que cabría haber esperado. 
En el periodo de crisis posterior la situación experimenta un cambio notable. La tasa estimada del crecimiento de la calidad del factor trabajo obtenida a partir de las técnicas de la contabilidad del crecimiento aumenta de ritmo. Su tasa de crecimiento prácticamente se duplica, situándose en torno al 1 por 100 anual, una cifra comparable a la mejora de las dotaciones educativas de la población ocupada durante esos años. La crisis habría tenido consecuencias claras en términos del uso del capital humano de la economía española, forzando un uso más eficiente de los factores productivos, en este caso del trabajo.

Los resultados obtenidos usando solo los datos salariales de 2002 o solo los datos salariales de 2006 son cualitativamente similares. Las diferencias, aunque no excesivas, reflejan una cierta tendencia a la moderación en la mejora de la calidad del trabajo. Esto hay que ligarlo a la caída de los rendimientos de la experiencia, la educación, etc., observados en el Cuadro 1.

Los problemas de sobreducación y excesiva temporalidad podrían estar detrás de esa insuficiente contribución a la productividad. A fin de considerar esa cuestión vamos a considerar escenarios alternativos suponiendo diferentes porcentajes de reducción en el peso de los trabajadores sobreducados y temporales al final de cada periodo respecto al dato real. Los resultados de esos ejercicios contrafactuales se ofrecen en el Cuadro 3.

\section{CUADRO 3}

EFECTOS DE LA SOBREDUCACIÓN Y LA TEMPORALIDAD SOBRE EL CRECIMIENTO DE LA CALIDAD DEL FACTOR TRABAJO (En \% anual)

\begin{tabular}{|c|c|c|c|}
\hline & & \multicolumn{2}{|c|}{ Reducción de un 10 por 100 de los.... } \\
\hline & Ecuaciones salariales & Sobreducados & \multicolumn{2}{|c|}{ Temporales } \\
\hline $2002-2006$ & 0,48 & 0,60 & 0,55 \\
\hline $2006-2010$ & 0,88 & 1,01 & 0,92 \\
\hline & & \multicolumn{2}{|c|}{ Reducción de un 20 por 100 de los... } \\
\hline & Ecuaciones salariales & Sobreducados & Temporales \\
\hline $2002-2006$ & 0,48 & 0,71 & 0,61 \\
\hline $2006-2010$ & 0,88 & 1,13 & 0,95 \\
\hline & & \multicolumn{2}{|c|}{ Reducción de un $\mathbf{5 0}$ por 100 de los... } \\
\hline & Ecuaciones salariales & Sobreducados & Temporales \\
\hline $2002-2006$ & 0,48 & 1,04 & 0,75 \\
\hline $2006-2010$ & 0,88 & 1,46 & 0,90 \\
\hline
\end{tabular}

NOTA: Contrafactuales obtenidos aplicando los salarios relativos estimados a partir de la EES-2002 en 2002 y de la EES-2006 a partir de 2006. 
La primera columna del cuadro 3 muestra los valores estimados para la evolución real de cada tipo de empleo y, por tanto, coinciden con los ya comentados en el Cuadro 2. El resto de columnas se refiere al hipotético crecimiento que habría sido estimado con las técnicas de contabilidad del crecimiento bajo diferentes supuestos acerca de la reducción de los problemas de sobreducación de los trabajadores con formación superior y de temporalidad. Esto es, se trata de ver el impacto de reubicar en el año final una parte de los trabajadores sobreducados (o temporales) en una categoría caracterizada por la misma edad, sexo, nivel educativo, etc., pero con una ocupación adecuadamente justificada (o con un contrato indefinido) ${ }^{4}$.

Como puede apreciarse, cuanto mayor es la mejora en esos ámbitos mayor es el impacto en términos de crecimiento de la productividad. Centrémonos en el caso de una mejora lo suficientemente intensa como para situar a España en los niveles habituales en la Unión Europea (esto es, aproximadamente una reducción a la mitad en la intensidad del problema).

Una reducción de la sobreducación hasta la media europea habría supuesto más que duplicar la mejora de calidad del factor trabajo y, por tanto, su contribución al crecimiento de la productividad. Durante la fase de expansión se habría alcanzado un crecimiento anual del 1,04 por 100 en vez del 0,48 por 100 estimado y durante la fase posterior de crisis el 1,46 por 100 en vez del 0,88 por 100 estimado.

En el caso de la temporalidad, una convergencia con la media de la UE habría implicado, durante el periodo expansivo, pasar al 0,75 por 100 anual y, durante el periodo 2006-2010, al 0,90 por 100 .

\section{Conclusiones}

La evolución de la productividad del trabajo durante las últimas décadas en España resulta poco compatible con las aparentes mejoras de la formación educativa de la población ocupada. Esto plantea dudas respecto a la rentabilidad del fuerte esfuerzo de inversión en capital humano que viene realizando nuestro país, esfuerzo compartido por familias y sector público. Además, tiene un impacto claramente negativo en la competitividad de la economía y en sus posibilidades de desarrollo futuro.

La educación y el capital humano son motores fundamentales del progreso de las sociedades una vez éstas han alcanzado un nivel avanzado de desarrollo como el logrado por España. Si estos motores no funcionan adecuadamente las perspectivas de futuro de una economía como la española se complican considerablemente.

En este trabajo se ha analizado la evolución en ese ámbito de España durante la última expansión y también en lo que llevamos de crisis. Para ello se han utilizado microdatos de la EPA y de sucesivas encuestas salariales y se han aplicado técnicas de

${ }^{4}$ El supuesto implícito es que ese cambio no afectaría a los salarios relativos de cada categoría. Cambios en esos salarios relativos afectarían a las estimaciones ofrecidas en este trabajo. 
contabilidad de crecimiento, algo que permite estudiar el impacto de los problemas de productividad planteados por la sobreducación de nuestros trabajadores más formados y por la excesiva temporalidad y su carácter generalizado. En esos dos aspectos se ha centrado el análisis.

Los resultados indican el negativo impacto de los excesivos niveles de desajuste educativo y de temporalidad, de modo muy especial durante la fase expansiva. Esto apunta al carácter poco sostenible y apenas preocupado por la eficiencia y la productividad (cuestiones clave a medio y largo plazo) de nuestro patrón de crecimiento pasado. Como resultado el efecto de la crisis ha sido especialmente duro en España, pero el análisis del periodo más reciente indica que los dolorosos ajustes llevados a cabo van en la dirección correcta para seguir, una vez superada la crisis, un tipo de crecimiento más sano y sostenible, más basado en el conocimiento y el uso apropiado del capital humano.

Los resultados obtenidos indican que una convergencia a los niveles europeos medios de sobreducación habría supuesto duplicar la contribución del factor trabajo al crecimiento de la productividad. Un progreso similar en términos de temporalidad también habría tenido un impacto sustancial, especialmente durante el periodo de fuerte creación de empleo previo a la crisis.

Todo esto apunta a la necesidad de redoblar los esfuerzos dirigidos a mitigar los problemas de desajuste educativo, una tarea que afecta tanto al sector público como al privado, tanto al sistema educativo como a las empresas. Del mismo modo, hay que explotar a fondo las posibilidades de la última reforma laboral para dejar atrás el abuso en la utilización de los contratos temporales. La mayor flexibilidad introducida por esa reforma en diferentes ámbitos del mercado de trabajo debería ser una pieza clave para mantener en el futuro, también en épocas de bonanza, un modelo de crecimiento sostenible y competitivo basado en las ganancias de productividad y en la calidad de los factores productivos y no tanto en la mera cantidad de los mismos.

\section{Referencias bibliográficas}

[1] ALBA-RAMÍREZ, A. (1993): «Mismatch in the Spanish Labor Market: Overeducation?», The Journal of Human Resources, 28, 259-278.

[2] BUDRÍA, S. y MORO-EGIDO, A. I. (2008): «Education, Over-education and Wage Inequality: Evidence for Spain», Economics of Education Review, vol. 27, pp. 332-341.

[3] CARRASCO, R.; JIMENO, J. F. y CAROLINA ORTEGA, A. (2011): «Accounting for changes in the Spanish wage distribution: the role of employment composition», Documento de Trabajo 1120, Banco de España.

[4] CUADRADO-ROURA, J. R. y MAROTO,A. (2012): El problema de la productividad en España: causas estructurales, cíclicas y sectoriales, FUNCAS.

[5] DE LA FUENTE, A. (2002): «On the sources of convergence: a close look at the Spanish regions», European Economic Review, 46 (3), 569-599.

[6] FELGUEROSO, F.; HIDALGO, M. y JIMÉNEZ-MARTÍN, S. (2010): «Explaining the fall of the skill wage premium in Spain», Documento de Trabajo FEDEA 2010-19. 
[7] GARCÍA-MONTALVO, J. y PEIRÓ, J. M. (2009): Análisis de la sobrecualificación y la flexibilidad laboral. Observatorio de la inserción laboral de los jóvenes 2008, Valencia, Fundación Bancaja.

[8] HERNÁNDEZ, L. y SERRANO, L. (2012): «Overeducation and its effects on wages: a closer look at the Spanish regions», Investigaciones Regionales, 24, 57-88.

[9] JORGENSON, D. W.; GOLLOP, F. M. y FRAUMENI, B.M. (1987): Productivity and U.S. Economic Growth, Cambridge, MA, Harvard University Press.

[10] LACUESTA, A.; PUENTE, S. y CUADRADO, P. (2011): «Omitted variables in the measurement of a labor quality index: the case of Spain», Review of Income and Wealth, 57 (1), 84-110.

[11] LUCAS, R. E. (1988): «On the mechanics of economic development», Journal of Monetary Economics, 22 (1), 3-42.

[12] MANKIW, N. G.; ROMER, P. y WEIL, D. (1992): «A contribution to the empirics of economic growth», Quarterly Journal of Economics, 107 (2), 407-437.

[13] MAS, M. y ROBLEDO, J. C. (2010): Productividad, una perspectiva internacional y sectorial, Bilbao, Fundación BBVA.

[14] MAS, M. y STEHRER, R. (2011): Industrial Productivity in Europe, Growth and Crisis, Cheltenham (UK) y Northampton (MA, Estados Unidos), Edward Elgar.

[15] MINCER, J. (1974): Schooling, experience and earnings, Nueva York, Columbia University Press.

[16] MURILLO, I. P.; RAHONA, M. y SALINAS, M. (2010): «Efectos del desajuste educativo sobre el rendimiento privado de la educación: un análisis para el caso español (1995-2006)», Documento de Trabajo n. ${ }^{\circ}$ 520, Fundación de las Cajas de Ahorros (FUNCAS).

[17] NELSON, R. y PHELPS, E. (1966): «Investments in humans, technological diffusion and economic growth», American Economic Review, Papers and Proceedings, 56 (2), 69-75.

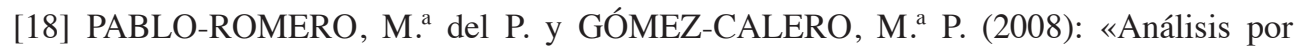
provincias de la contribución del capital humano a la producción en la década de los noventa», Investigaciones Económicas, 32 (1), 27-52.

[19] PASTOR, J. M.; RAYMOND, J.L.; ROIG, J.L. y SERRANO, L. (2007): El rendimiento del capital humano en España, Fundación Bancaja, Valencia.

[20] RAYMOND, J. L. (coord.) (2012): «¿Es rentable educarse? Marco conceptual y principales experiencias en los contextos español, europeo y en países emergentes». Estudios de la Fundación. Economía y Sociedad, n. ${ }^{\circ}$ 53, Fundación de las Cajas de Ahorros (FUNCAS).

[21] ROMER, P. M. (1990): «Endogenous technological change», Journal of Political Economy, 98, S7-S102.

[22] SCHULTZ, T. (1960): «Capital formation by education», Journal of Political Economy, 69, 571-83.

[23] SERRANO, L. (1999): «Capital humano, estructura sectorial y crecimiento en las regiones españolas», Investigaciones Económicas, 23 (2), 225-250.

[24] SERRANO, L. (2012): «Resultados educativos y crecimiento económico en España», en A. Villar (dir.), Educación y desarrollo. PISA 2009 y el sistema educativo español, Fundación BBVA. 
[25] SERRANO, L. y SOLER, A. (2010): Metodología para la estimación de las Series de Capital Humano 1964-2010, Fundación Bancaja-Ivie. (Series de capital humano 19642010 disponibles en Internet: http://www.ivie.es/banco/caphumser10.php).

[26] SOSVILLA-RIVERO, S. y ALONSO MESEGUER, J. (2005): «Estimación de una función de producción MRW para la economía española 1910-1995», Investigaciones Económicas, 29 (3), 609-624.

[27] WELCH, F. (1970): «Education in production», Journal of Political Economy, 78, 35-59. 